\title{
Analytical and experimental pharmacology, challenges ahead
}

\author{
Salvatore Salomone* \\ Department of Pharmacology, Catania University, Catania, Italy \\ *Correspondence: salomone@unict.it
}

Everybody would agree in saying that advances in Medicine, in the last decades, have lead to significant increase in life expectancy. However, the direct impact of global drug development might be harder to be precisely defined. Let then consider the following example. In the years between 1990 and 1994 AIDS was the first cause of death among people $<45$ in USA, with up to 40 deaths/year/100,000. In 1996-1998, AIDSrelated deaths dropped sharply, far below cancer and cardiovascular deaths, down to $10 /$ year/100,000, as a result of approval by FDA of novel drugs to treat HIV infection, such as protease inhibitors. Here a striking example of the impact of drug discovery on life expectancy. But where did these novel drugs come from? At variance with reverse transcriptase inhibitors, the discovery of first HIV protease inhibitors did not come from massive screening of randomly generated chemical libraries, but rather from a rational process, based on detailed study of proteolytic mechanisms in vertebrates and in HIV, analysis of differences in these processes, drug design based on transition state mimetic concept, an approach that had already been successfully used in the design of inhibitors of other aspartic proteinases such as renin (Roberts et al., 1990). This example tells us two important things: (1) drug discovery may rapidly and dramatically change every day life; (2) innovative drugs may be obtained through rational, analytical research.

\section{WHAT DO WE MEAN BY "ANALYTICAL AND EXPERIMENTAL" PHARMACOLOGY?}

We may propose that it is the analytical study of the mechanism of drug action, at the molecular, cellular and organism levels. This involves in silico analysis, quantitative structure-activity relationship (QSAR), drug design, as well as investigation on experimental drugs (compounds acknowledged as useful research tools, but not intended for human use) or further investigation on drugs approved and already in use. Conceptually, the first, lower level of analysis is the molecular one, which is the interaction of the drug with biomolecular targets such as, in many instances, receptors. This seems obvious to us, but certainly was not obvious in the 50s, when, for example, the study of pharmacology of catecholamines was moving its first steps. At that time, very few people in the world were using the term "receptor" and none of them had any idea about the physical entity they were dealing with (a transmembrane protein, product of gene expression). The most strenuous defender and propagator of that concept, Ahlquist, thought, in fact, that receptor was an idea, useful to explain the physiological effects of adrenaline and isoprenaline on cardiovascular and respiratory systems, rather than a physical object interacting with hormones and drugs (Ahlquist, 1973). Nevertheless, this way of thinking (and acting) about receptors, affinity, efficacy, agonists, antagonists, etc... produced the paradigm that founded the modern pharmacology and impressed a robust momentum to drug discovery and development of pharmaceutical industry. In this pioneering time, the technology available to any potential pharmacologist was minimal; no commercial fine chemistry, no commercial machines, no computers, very limited funding either governmental/no-profit or private/corporate. Drug discovery often moved from serendipitous observations or, less often, from existing endogenous biomolecules, used as lead compounds for synthesizing new drugs, as, for example, Black et al. (1965) did for beta-blockers. The availability of these new selective agents further drove the research to understand receptor distribution, subtypes, physiological effects related to their stimulation. At that time the analysis of interaction between drug and receptor was conducted at the organism level (blood pressure, heart rate, glucose, and electrolytes in blood and urine) or at the organ level (isolated vessels, isolated whole heart/Langendorf, isolated papillary muscle, isolated bronchi). The pharmacological analysis barely involved the cell level. In the 70s, commercial availability of devices for measuring beta and gamma emission together with commercial availability of radioisotopes and/or radioligands (drugs bearing radioactive isotopes in their molecule) permitted systematic and quantitative studies of drug receptor binding, demonstrating the physical existence of receptors as discrete entities, showing their precise distribution, measuring their abundance and affinity. Until this time, the analysis of drug effects was still carried out at the organism/organ level. The goals of drug discovery (and of Pharmacology) were therefore focused on obtaining tools for changing physiological parameters which would possibly positively influence the disease outcome and/ or relieve/palliate some symptoms. Very little was known about the fundamental mechanisms of major human chronic diseases (cardiovascular, diabetes, cancer), the cellular and the molecular level of analysis remained off limits for Pharmacology. The goal of developing and implementing anti-infectious agents (antibiotics), since the $40 \mathrm{~s}$, and anticancer drugs, since the $60 \mathrm{~s}$, oriented pharmacological research toward the cellular and the molecular levels. The major parameter of drug efficacy considered in this context was cell proliferation, but relevant was also the analysis of metabolic bacterial pathways at the biochemical level. In the meanwhile, electrophysiology was bursting neuroscience, demonstrating the electrical/chemical coupling in central and peripheral nervous system, i.e., the effect of neurotransmitters (and/or their synthetic counterparts, agonists and antagonists) at the cellular level (action potential, IPSP, and EPSP) in the 60s (Eccles and Liley, 1959; Eccles et al., 1963), and at the molecular level (i.e., single channel record) in the 70s (Neher and Sakmann, 
1976). The true paradigm shift in life sciences, however, occurred in the $80 \mathrm{~s}$, when rapid advances in molecular biology produced novel knowledge and novel technology that spread all around the world. This shift was founding an ultimate ambitious reductionism, still prevalent in now days, stating that all kind of life may be understood (and manipulated) as information encoded in biomolecules (DNA, RNA, proteins). The analysis of mechanisms of drug action therefore has definitively advanced to the molecular level. The paradigm shift represented by the biomolecular reductionism, has also changed the path to drug discovery, and its impact in every-day drug therapy is continuously and rapidly growing.

\section{RANDOM SCREENING AND CONCEPT- DRIVEN SCREENING}

In its beginnings, drug discovery was slowly progressing, based mainly on tradition and serendipity, i.e., systematic pharmacological analysis of traditional remedies or fortuitous discovery of new effects. In few cases, when concept-driven, the discovery of new drugs was guided by analysis of "endogenous leads" (as mentioned above for beta-blockers). More recently, since the 70s, drug discovery had moved from the analysis of a known target (a receptor) to the random screening of numerous candidate compounds potentially binding the target (agonist, antagonists, etc...) and thereby modifying a given physiological function. Combinatorial chemistry provides, by random synthesis, large sets of compounds (libraries). Robotics enable routinely production of over 100,000 new and unique compounds per year. In order to handle the vast number of structural possibilities, researchers often create in silico libraries, consisting of thousands to millions of "virtual" compounds; selected subsets of the "virtual library" may eventually undergo actual synthesis, based upon further analysis (pharmacokinetics, computational chemistry, and QSAR). Although combinatorial chemistry has been an essential part of drug discovery, a large proportion of new chemical entities still are nature-derived compound. As Feher and Schmidt (2003) pointed out, this may be in part related to the fact that combinatorial libraries lack chirality and structure rigidity, the two most important features distinguishing approved drugs and natural products from randomly synthesized combinatorial compounds. High-throughput screening (HTS) is the method that allows a researcher to conduct as many as millions of biological tests. HTS uses automation to screen libraries of candidate compounds against targets, looking for specific activity such as inhibition or stimulation of defined biological mechanisms. Microtiter plates, filled with nanoliters of solution containing the reactants, are incubated and then read by automated analysis machines. Current HTS robots can test up to 100,000 compounds per day, but higher performance systems are also available. Recently, an HTS process that uses drop-based microfluidics, allowing 100 million reactions in $10 \mathrm{~h}$, has been reported (Agresti et al., 2010).

Back in the 80s, it became clear that deciphering the entire genome of organisms was possible and that sequencing human genome, for example, was a valuable task deserving significant resources. This view lead to complete sequencing of human genome in 2000. Increasing availability of information and technology made the new paradigm applicable to all fields of life science, including those with practical significance for every day needs, such as medicine and agriculture. The enormous and continuously growing amount of data generated in the last 20 years, by systematic study of the molecular mechanisms in cardiovascular diseases, diabetes, cancer, permitted an understand of the molecular basis of diseases. The goals of drug discovery and pharmacology become therefore focused on obtaining tools for modifying specific molecular targets, possibly leading to definitive cure of the disease. Sometimes, it is possible to make specific prediction about levels of gene expression in disease; but more commonly, expression profiling takes place before enough is known about how genes interact with experimental conditions for a testable hypothesis to exist. In some of these cases, expression profiling helps to identify candidate genes relevant for mechanisms of disease and/or novel potential drug targets. Molecular analysis at the concept level (molecular mechanism of the disease, potential drug target), followed by drug design and experimental validation of drug efficacy, therefore starts flowing from technologies that allow large, comprehensive screening of information carried by biomolecules. This ambition is summarized in the -ome paradigm: genome, transcriptome, proteome, indicate complete sets of categories (genes, transcripts, proteins) accessible to direct scrutiny. Gene expression profiling measures the expression level of thousands of genes at once, creating a global picture of cell function. These profiles enable one to distinguish, for example, between normal differentiated quiescent cells in a control sample from those transformed in a tumor specimen; furthermore, gene expression profiling may show if cancer cells respond to a particular drug treatment or develop drug resistance, by changing the expression of genes involved in apoptosis, cell cycle, etc... DNA Microarray technology measures the relative activity of previously identified target genes, whereas sequence based techniques, like serial analysis of gene expression (SAGE, SuperSAGE) can accurately measure any active gene, not just a predefined set. More recently, human genome-wide association studies deal with complex traits that consist of a limited number of common alleles, each conferring a small increase in risk to the individual. A substantial proportion of the human genome is comprised of regions that exhibit reduced recombination in human populations (haplotypes). Genetic variants within haplotypes are in linkage disequilibrium. Several million single nucleotide polymorphisms (SNPs) have been identified and genome-wide maps of SNP are available. These resources, together with platforms for massive SNP genotyping, have rendered genome-wide association studies feasible for diseases such as diabetes mellitus (Saxena et al., 2007), myocardial infarction (Mizuno et al., 2006), cancer (Gudmundsson et al., 2007), inflammatory bowel disease (Xavier and Podolsky, 2007).

Other techniques, like the two-hybrid screening (also known as yeast two-hybrid system or $\mathrm{Y} 2 \mathrm{H}$ ), allow massive analysis of protein-protein interactions or proteinDNA interactions (for DNA-binding proteins, such as transcription factors). These technologies have lead to the constructions of interactomes, i.e., maps of all the protein- 
protein or protein-DNA interactions in different species, including human. Analysis of interactomes are increasingly proven useful in studying signaling pathways downstream receptor activation, including kinases involved in receptor phosphorylation and down-regulation.

\section{ANALYTICAL PHARMACOLOGY FOR DRUGS OF 21ST CENTURY}

A significant proportion of newly approved drugs (over 25\%) is now days represented by biologicals (or biotechnology-derived pharmaceuticals); many of them are monoclonal antibodies, often intended for cancer treatment, other are DNA and RNA species (including siRNA), intended for acting in a sequence-specific manner (i.e., the Watson and Crick base pairing is exploited to obtain drug-specificity). For these new drug types new categories of analysis are required. The regulatory agencies are setting specific rules for approval, though most of the classical preclinical pharmacological and toxicological analysis remain mandatory. Biological activity may be evaluated using in vitro assays, including cell lines and/or primary cell cultures to examine the direct effects on cellular phenotype and proliferation; in vitro studies are also designed to determine receptor occupancy and receptor affinity. Due to the species specificity of many biologicals, it is important to select relevant animal species for in vivo testing. Large gene expression profiling may become essential not only for showing (or excluding) off target effects, but also for understanding complex in vivo effects, resulting from modification of expression of multiple genes. Different issues may apply to aptamers, DNA- or RNA-related compounds that exert biological actions not through the information encoded in their sequence, but by specifically interacting with biomolecules (somehow like the classical chemically synthesized drugs). The mechanism of action of these agents might still be analyzed in terms of QSAR, affinity, efficacy. This field is likely to substantially grow in the next years, because patents restricting the diffusion of the aptamerrelated technology are expiring. Specific issues, however, may concern the pharmacokinetics of aptamers, their molecular size being usually bigger than classical, chemically synthesized, drugs.

\section{WHAT KIND OF PHARMACOLOGICAL ANALYSIS DO WE NEED TODAY?}

Molecular analysis is essential in drug design and discovery; this is mostly carried out on lead compounds, either derived from random HTS or from concept-driven identification of molecular target (gene profiling analysis followed by in silico QSAR and drug synthesis).

Cellular analysis is essential for assessing the consequences of drug-target interaction on cell physiology and signaling (generation of second messengers, calcium release from endoplasmic reticulum, calcium waves and spikes, electrical phenomena, IPSP, EPSP, action potential, consequences of kinase or phosphatase inhibition on downstream signaling, activation and inhibition of transcription factors and impact on gene expression, cell cycle and proliferation, cell differentiation and apoptosis).

Organism analysis is essential for demonstrating the drug effect(s) that are exploitable to cure diseases or relieve symptoms. In most instances this cannot be deduced solely by analysis at the molecular and/ or cellular level, because the mechanism of the disease (and of the cure) operate exclusively at the organism level. Here few examples: diseases like migraine, epilepsy, brain ischemia, asthma, heart failure, liver cirrhosis; symptoms like tremor, amnesia, hypertension, vomits, fever, diarrhea.

These three fundamental levels of analysis today are not dispensable when developing new therapeutics, but can also be applied to already existing and approved drugs, to unravel novel indications and/or to predict potential harmful effects.

Finally, a further, more recently introduced, fourth level of analysis is that of individualized pharmacology, i.e., the analysis of the effect of genetic variation on drug response in patients, by correlating gene expression level and/or SNPs with drug efficacy or toxicity. The challenge introduced by pharmacogenomic analysis is to optimize drug therapy for each individual's unique genetic makeup, ensuring maximum efficacy while minimizing adverse effects.

\section{REFERENCES}

Agresti, J. J., Antipov, E., Abate, A. R., Ahn, K., Rowat, A. C., Baret, J. C., Marquez, M., Klibanov, A. M., Griffiths, A. D., and Weitz, D. A. (2010). Ultrahighthroughput screening in drop-based microfluidics for directed evolution. Proc. Natl. Acad. Sci. U.S.A. 107, 4004-4009.

Ahlquist, R. P. (1973). Adrenergic receptors: a personal and practical view. Perspect. Biol. Med. 17, 119-122.

Black, J. W., Duncan, W. A., and Shanks, R. G. (1965). Comparison of some properties of pronethalol and propranolol. Br. J. Pharmacol. Chemother. 25, 577-591.

Eccles, J. C., and Liley, A. W. (1959). Factors controlling the liberation of acetylcholine at the neuromus- cular junction. Am. J. Phys. Med. 38, 96-103.

Eccles, J. C., Schmidt, R., and Willis, W. D. (1963). Pharmacological studies on presynaptic inhibition. J. Physiol. $168,500-530$.

Feher,M., andSchmidt,J.M.(2003).Property distributions: differences between drugs, natural products, and molecules from combinatorial chemistry. J. Chem. Inf. Comput. Sci. 43, 218-227.

Gudmundsson,J.,Sulem,P.,Manolescu,A., Amundadottir, L. T., Gudbjartsson, D., Helgason, A., Rafnar, T., Bergthorsson, J. T., Agnarsson, B. A., Baker, A., Sigurdsson, A., Benediktsdottir, K. R., Jakobsdottir, M., Xu, J., Blondal, T., Kostic, J., Sun, J., Ghosh, S., Stacey, S. N., Mouy, M., Saemundsdottir, J., Backman, V. M., Kristjansson, K., Tres, A., Partin, A. W., Albers-Akkers,
M. T., Godino-Ivan, M. J., Walsh, P. C., Swinkels, D. W., Navarrete, S., Isaacs, S. D., Aben, K. K., Graif, T., Cashy, J., Ruiz-Echarri, M., Wiley, K. E., Suarez, B. K., Witjes, J. A., Frigge, M., Ober, C., Jonsson, E., Einarsson, G. V., Mayordomo, J. I., Kiemeney, L. A., Isaacs, W. B., Catalona, W. J., Barkardottir, R. B., Gulcher, J. R., Thorsteinsdottir, U., Kong, A., and Stefansson, K. (2007). Genome-wide association study identifies a second prostate cancer susceptibility variant at 8q24. Nat. Genet. 39, 631-637.

Mizuno, H., Sato, H., Sakata, Y., Ohnishi, Y., Hishida, E., Kinjo, K., Nakatani, D., Shimizu, M., Kondo, H., Tanaka, T., Ozaki, K., Hirayama, A., Ito, H., Otsu, K., and Hori, M. (2006). Impact of atherosclerosis-related gene polymorphisms on mortality and recur- rent events after myocardial infarction. Atherosclerosis 185, 400-405.

Neher,E., and Sakmann, B. (1976). Singlechannel currents recorded from membrane of denervated frog muscle fibres. Nature 260, 799-802.

Roberts, N. A., Martin, J.A., Kinchington, D., Broadhurst, A. V., Craig, J. C., Duncan, I. B., Galpin, S. A., Handa, B. K., Kay, J., Krohn, A., Lambert, R. W., Merrett, J. H., Mills, J. S., Parkes, K. E. B., Redshaw, S., Ritchie, A. J., Taylor, D. L., Thomas, G. J., and Machin, P. J. (1990). Rational design of peptidebased HIV proteinase inhibitors. Science 248, 358-361.

Saxena, R., Voight, B. F., Lyssenko, V., Burtt, N. P., de Bakker, P. I., Chen, H., Roix, J. J., Kathiresan, S., Hirschhorn, J. N., Daly, M. J., Hughes, T. E., Groop, L., Altshuler, D., Almgren, P., Florez, J. 
C., Meyer, J., Ardlie, K., Bengtsson, B. K., Isomaa, B., Lettre, G., Lindblad, U., Lyon, H. N., Melander, O., NewtonCheh, C., Nilsson, P., Orho-Melander, M., Råstam, L., Speliotes, E. K., Taskinen, M. R., Tuomi, T., Guiducci, C., Berglund, A., Carlson, J., Gianniny, L., Hackett, R., Hall, L., Holmkvist, J., Laurila, E., Sjögren, M., Sterner, M., Surti, A., Svensson, M., Svensson, M., Tewhey, R., Blumenstiel, B., Parkin,
M., Defelice, M., Barry, R., Brodeur, W., Camarata, J., Chia, N., Fava, M., Gibbons, J., Handsaker, B., Healy, C., Nguyen, K., Gates, C., Sougnez, C., Gage, D., Nizzari, M., Gabriel, S. B., Chirn, G. W., Ma, Q., Parikh, H., Richardson, D., Ricke, D., and Purcell, S. (2007). Genome-wide association analysis identifies loci for type 2 diabetes and triglyceride levels. Science 316, 1331-1336.
Xavier, R. J., and Podolsky, D. K. (2007). Unravelling the pathogenesis of inflammatory bowel disease. Nature 448, 427-434.

Received:06August 2010; accepted:31 August 2010; published online: 17 September 2010. Citation: Salomone S (2010) Analytical and experimental pharmacology, challenges ahead. Front. Pharmacol. 1:119. doi: 10.3389/fphar.2010.00119
This article was submitted to Frontiers in Experimental Pharmacology and Drugs Discovery, a specialty of Frontiers in Pharmacology.

Copyright (c) 2010 Salomone. This is an open-access article subject to an exclusive license agreement between the authors and the Frontiers Research Foundation, which permits unrestricted use, distribution, and reproduction in any medium, provided the original authors and source are credited. 